APS

physics

This is the accepted manuscript made available via CHORUS. The article has been published as:

\title{
Nontrivial Berry phase and type-II Dirac transport in the layered material PdTe_\{2\}
}

Fucong Fei, Xiangyan Bo, Rui Wang, Bin Wu, Juan Jiang, Dongzhi Fu, Ming Gao, Hao Zheng, Yulin Chen, Xuefeng Wang, Haijun Bu, Fengqi Song, Xiangang Wan, Baigeng Wang, and Guanghou Wang

Phys. Rev. B 96, 041201 - Published 5 July 2017

DOI: 10.1103/PhysRevB.96.041201 


\section{Nontrivial Berry Phase and Type-II Dirac Transport in Layered Material PdTe}

Fucong $\mathrm{Fei}^{1}$, Xiangyan $\mathrm{Bo}^{1}$, Rui Wang ${ }^{1}$, Bin $\mathrm{Wu}^{1}$, Juan Jiang ${ }^{2}$, Dongzhi Fu ${ }^{1}$, Ming $\mathrm{Gao}^{3}$, Hao Zheng ${ }^{4}$, Yulin Chen ${ }^{2,5}$, Xuefeng Wang ${ }^{3}$, Haijun Bu ${ }^{1}$, Fengqi Song ${ }^{1} *$, Xiangang Wan ${ }^{1, \dagger}$, Baigeng Wang ${ }^{1, \ddagger}$, Guanghou Wang ${ }^{1}$

${ }^{1}$ National Laboratory of Solid State Microstructures, Collaborative Innovation Center of Advanced Microstructures, and College of Physics, Nanjing University, Nanjing, 210093, P. R. China

${ }^{2}$ School of Physical Science and Technology, ShanghaiTech University, and CAS-Shanghai Science Research Center, Shanghai 200031, P. R. China

${ }^{3}$ National Laboratory of Solid State Microstructures, Collaborative Innovation Center of Advanced Microstructures, and School of Electronic Science and Engineering, Nanjing University, Nanjing, 210093, P. R. China

${ }^{4}$ Department of Physics and Astronomy, Shanghai Jiao Tong University, Shanghai 200240, P. R. China

${ }^{5}$ Department of Physics, University of Oxford, Oxford, OX1 3PU, UK 


\begin{abstract}
We report on a systematic study of type-II Dirac fermions in a layered crystal of $\mathrm{PdTe}_{2}$. De Haas-van Alphen oscillations show a small Fermi surface pocket with a cross section of $0.077 \mathrm{~nm}^{-2}$ with a nontrivial Berry phase. First principles calculation reveals that the nontrivial Berry phase originates from a hole pocket formed by the tilted Dirac cone. In addition, the band dispersion measured with angle resolved photoemission spectroscopy is found to be consistent with that of a type-II Dirac cone dispersion. We propose that $\mathrm{PdTe}_{2}$ is an improved platform to host topological superconductors.
\end{abstract}




\section{INTRODUCTION}

In condensed matters, multiple excitations can be implemented to simulate the physics of new particles, such as Dirac fermions [1-10], Weyl fermions [11-24], Majorana fermions [25-28] and exotic new fermions beyond Dirac and Weyl fermions [29] with the potential applications in next-generation spintronics and quantum computing. These particles might be unrealistic in real world while allowable in condensed matters due to versatile operations of symmetry breaking. In Dirac semimetals, e. g., $\mathrm{Na}_{3} \mathrm{Bi}$ [8-10] and $\mathrm{Cd}_{3} \mathrm{As}_{2}$ [1-7] and topological insulators [30-33], topological protected gapless Dirac cones with liner dispersions are discovered and electrons in these materials can be described by massless Dirac equation. By breaking inversion symmetry or time reversal symmetry, a Dirac cone can split into a pair of Weyl cones and form Weyl semimetals such as TaAs family [12-17]. Noncontinuous Fermi arcs appear on their surfaces and interesting negative magnetoresistance appears, which simulates the chiral anomaly in high energy physics. In several Weyl semimetals, for instance $\mathrm{Mo}_{\mathrm{x}} \mathrm{W}_{1-\mathrm{x}} \mathrm{Te}_{2}$ [19-23] and LaAlGe [11], the Lorentz invariance is broken and the Weyl cones are strongly tilted to form type-II Weyl semimetals, which are predicted to have unique properties such as field-selective anomaly and chiral mode reversal [34], as well as Klein tunneling in momentum space [35]. Recently, the Lorentz invariance breaking is suggested in Dirac semimetals with the result of type-II Dirac fermions with tilted Dirac cone in $\mathrm{PtSe}_{2}$ family [36-38].

Here we report the Shubnikov-de Haas ( $\mathrm{SdH})$ and de Haas-van Alphen (dHvA) 
oscillations in a layered material $\mathrm{PdTe}_{2}$ and a nontrivial Berry phase is demonstrated for the first time. Six conductive pockets are identified in the dHvA measurements, where the $\alpha$ mode with the frequency of $8.0 \mathrm{~T}$ exhibits the nontrivial Berry phase. The calculations confirm the Berry phase is originated from the hole pocket of a tilted type-II Dirac cone. This is also confirmed by the dispersions in Angle Resolved Photoemission Spectroscopy (ARPES). Remembering its anisotropic superconductivity under $1.9 \mathrm{~K}$, we suggest $\mathrm{PdTe}_{2}$ might be an improved platform to host the topological superconductors (TSCs).

\section{EXPERIMENT}

Single crystal $\mathrm{PdTe}_{2}$ was grown by the melt-growth method. A certain amount of Pd and Te powder (from Alfa Aesar) with atomic ratio 1:2.2 was sealed in an evacuated ampoule. The ampoule was heated up and maintained at $790^{\circ} \mathrm{C}$ for 48 hours. The melt was then slowly cooled down to $500^{\circ} \mathrm{C}$ in 7 days and then annealed at this temperature for 7 more days before natural cooling to room temperature. Millimeter-sized crystals with metallic luster were obtained, which could be exfoliated to small flakes by a knife easily. As shown in Fig. 1(a), $\mathrm{PdTe}_{2}$ is a type of layered transition metal dichalcogenides (TMD) material of $\mathrm{CdI}_{2}$-type structure with the $P \overline{3} m 1$ space group. The $\mathrm{x}$-ray diffraction data of the single crystal is shown in Fig. 1(b). The strong (00n) peaks of $\mathrm{PdTe}_{2}$ can be clearly seen and no other impurity peaks can be found, indicating nice crystallization of the $\mathrm{PdTe}_{2}$ sample. Fig. 1(c) shows the energy dispersive spectra for a typical $\mathrm{PdTe}_{2}$ flake. The ratio between $\mathrm{Pd}$ and Te elements is 1:1.99, indicating the stoichiometric ratio of the sample. The EDS 
mapping (insets of Fig. 1(c)) show the uniform elemental distribution of $\mathrm{Pd}$ and $\mathrm{Te}$, respectively. Fig. 1(d) is the Raman spectrum and shows two peaks at 74.1 and 131.5 $\mathrm{cm}^{-1}$ respectively.

\section{RESULTS AND DISCUSSION}

\section{A. Electrical transport properties}

We measure the magnetoresistance (MR) of $\mathrm{PdTe}_{2}$ single crystal up to $33.5 \mathrm{~T}$ under 1.7K. The longitudinal resistance is shown in Fig. 2(a) and $\mathrm{SdH}$ oscillations can be identified above $15 \mathrm{~T}$ as seen in the inset, indicating the high quality of the crystal. Fig. 2(b) shows the hall resistance under various temperatures from $2 \mathrm{~K}$ to $150 \mathrm{~K}$. One can clearly see the hall resistance versus magnetic field is linear at $150 \mathrm{~K}$ but becomes bended when the temperature goes down to $2 \mathrm{~K}$, indicating the multiple band transport with complex contribution from various conductive pockets near the Fermi surface [39].

$\mathrm{PdTe}_{2}$ is a superconductor with $T_{c} \sim 2 \mathrm{~K}$ [40]. We make further study on the superconductivity property of $\mathrm{PdTe}_{2}$. The crystal shows an anisotropic superconductivity as seen when we measure the temperature dependent resistance under magnetic field using two different sets of devices of configuration 1 (the current is perpendicular to c axis; the field is parallel to c axis) and 2 (the current is parallel to c axis; the field is parallel to c axis), as seen in Fig. 2(c) and (d) respectively. Using configuration 2, with increasing the field to $500 \mathrm{Oe}$, the superconductivity is nearly suppressed while the superconductivity survives the field of 2000 Oe in configuration 
1. We can also see that in Fig. 2(d), the resistance increases when temperature goes down near $T_{c}$, which is absent in Fig. 2(c). The anisotropic superconductivity in $\mathrm{PdTe}_{2}$ is thus obvious.

\section{B. dHvA oscillations and nontrivial Berry phase}

The nontrivial Berry phase is demonstrated by the dHvA measurements after we measure the magnetization condition of the $\mathrm{PdTe}_{2}$ flakes under low temperatures. Fig. 3(a) displays the magnetization versus magnetic field $(\mathrm{B} / / \mathrm{c})$ for $\mathrm{PdTe}_{2}$ at $1.8 \mathrm{~K}$. Beautiful dHvA oscillations can be seen from the raw data of M-H curves. Fig. 3(b) shows the magnetization strength versus 1/B under various temperatures after the background subtraction. Multiple sets of oscillations, with the frequencies of $8.0(\alpha)$, $113.2(\beta), 117.9(\gamma), 124.3(\delta), 133.9(\varepsilon)$ and $455.8 \mathrm{~T}(\zeta)$, can be extracted from the M-H data by the fast Fourier transformation (FFT) (inset in Fig. 3(c)). The dHvA oscillation can be described by the Lifshitz-Kosevich (LK) formula [41]:

$$
\Delta M \propto-R_{T} R_{D} \sin \left[2 \pi\left(\frac{F}{B}-\left(\frac{1}{2}-\phi\right)\right)\right]
$$

The thermal damping factor is $R_{T}=\frac{\chi T}{\sinh (\chi T)}$ and the Dingle damping factor is $R_{D}=\exp \left(-\chi \cdot T_{D}\right)$ where $\chi=\frac{2 \pi^{2} k_{B} m^{*}}{\hbar e B} . \Phi$ is the phase shift and $\phi=\frac{\phi_{B}}{2 \pi}-\delta$, where $\Phi_{B}$ is the Berry phase and $\delta$ equals 0 and $\pm 1 / 8$ for two dimensional and three dimensional systems respectively. The effective mass $m^{*}$ can be extracted by the fit of the temperature dependence of the corresponding oscillation amplitude to the $R_{T}$. The Dingle temperature $T_{D}$ can also be obtained through the fit of Dingle damping factor $R_{D}$, meanwhile, quantum relaxation time $\tau$ can be calculated by $\tau=\frac{\hbar}{2 \pi k_{B} T_{D}}$ and 
quantum mobility $\mu_{q}=e \tau / m^{*}$. The Berry phases determine the topological properties of the conductive pockets and can be extracted using Landau level fan diagram. In dHvA oscillation, the integer landau index should be assigned when density of states (DOS) of Fermi surface, which is proportion to $\mathrm{d} M / \mathrm{d} B$, reaches a minimum. Therefore, the landau index of the dHvA oscillation minima should be $n-1 / 4$ [42]. We find $\alpha$ mode can be separated easily, while the four mid-frequency modes $(\beta, \gamma, \delta, \varepsilon)$ are with similar frequencies. In addition, oscillation of high-frequency mode $(\zeta)$ is masked by other oscillations because of the small amplitude. Therefore, we fit the oscillatory components of these five modes by the multiband LK formula, as shown in Fig. 3(e), and extract the transport parameters. All six sets of data of the dHvA oscillations are displayed in Table I.

Interestingly, a topological-nontrivial mode, the $\alpha$ mode, is evidenced by the Landau fan diagram, by using dHvA oscillation minima as the landau index of $n-1 / 4$, as shown in Fig. 3(d). The intercept of the linear fitting is 0.46 , which is the signature of nontrivial Dirac transport. Fig. 3(c) displays the fits of effective mass of the all six oscillatory modes by the corresponding FFT amplitudes versus temperature (inset in Fig. 3(c)). It is clear that the effective mass of the low-frequency mode $(\alpha)$ is much less than other modes. The low effective mass also agrees with the Dirac nature of the $\alpha$ mode.

\section{Theoretical calculation of band structures}


To identify the contributing electronic pocket of each oscillation mode, the assistance from the band structural calculation is needed. We calculate the band structure of $\mathrm{PdTe}_{2}$ (Fig. 4(a)) and the Brillouin zone (BZ) is shown in the inset of Fig. 1(a), where high-symmetry points, lines and Dirac point (D) are also indicated. There is a band crossing feature near the Fermi level along the $\Gamma$-A line. This band crossing is unavoidable, because these two bands belong to different representations (G4 and G5+G6 respectively). This is determined by the C3 rotational symmetry around the c axis $[36,37,43]$. Remarkably, these two bands show linear dispersions in the vicinity of the Dirac point along both the in-plane $\left(\mathrm{k}_{\mathrm{x}}-\mathrm{k}_{\mathrm{y}}\right)$ and out-of-plane $\left(\mathrm{k}_{\mathrm{z}}\right)$ directions and the Dirac cone is untilted along $\mathrm{k}_{\mathrm{x}}-\mathrm{k}_{\mathrm{y}}$ plane (Fig. $4(\mathrm{~d})$ ) but tilted strongly along $\left(\mathrm{k}_{\mathrm{z}}\right)$ direction (Fig. 4(e)), which is the characteristic feature of the type-II Dirac fermions as reported [36, 37]. By our calculations, the Dirac point is at $\mathrm{k}=(0,0, \pm 0.40)$.

In the calculation, a series of electronic pockets can be seen on the Fermi surface as shown in Fig. 4(b) and (c). For figuring out the contributing pocket of each dHvA oscillation mode, we calculate the extremal surfaces of each pocket along $\mathrm{k}_{\mathrm{z}}$ direction. The modes around $100 \mathrm{~T}$ were considered contributing from the minimum extremal surfaces (yellow area in Fig. 4(b)) of six pliers shaped pockets (translucent blue pockets in Fig. 4(b)) at $k_{z}= \pm 0.5$. Departure from high symmetry points and complex contours of these pockets may explain the several beat frequencies around 100T. The extremal surface of purple colored pocket at K point in Fig. 4(b) is about $0.04 \AA^{-2}$, which is the possible origin of $\zeta$ mode (456T). 
In type-II Dirac semimetals, the Dirac cone is tilted strongly because of the Lorentz invariance breaking, which causes the formation of a pair of electron and hole pockets[44]. In $\mathrm{PdTe}_{2}$, the Fermi level is above the Dirac point. Thus the hole pocket shrinks to a small pocket at $\Gamma$ point, as seen in Fig. 4(a) (also marked by red dash line in Figs. 4(b, c)) while the electron pocket, which corresponding to the translucent-aqua-like pocket in Figs. $4(\mathrm{~b}, \mathrm{c})$, becomes much bigger and forms an apple-pit-shaped pocket in reciprocal space. We find that the cut of the small hole pocket is with the similar area to that of the nontrivial $\alpha$ mode $(8 \mathrm{~T})$, which further confirms the Dirac nature of this pocket. For the big electron pocket, though the shape is complex, we verify that only a single maximum cross section achieves when $\mathrm{k}_{\mathrm{z}}=0$ after precise analysis. The corresponding oscillation frequency contribution is over $5 \mathrm{kT}$, which is too high to detect in our experiment.

\section{ARPES measurement of PdTe 2 samples}

The type-II Dirac dispersion in $\mathrm{PdTe}_{2}$ is characterized by the ARPES measurement.

Fig. 5(a) shows the electronic dispersion along T-D direction. The linear dispersion of Dirac cone can be clearly seen. Noting that T-D line is perpendicular to $k_{z}$, the Dirac cone is not tilted as predicted. The evolution of the Dirac cone dispersion can be clearly seen under different photon energies (Supplemental Material, Figure S1 [45]) which reveals the bulk properties of the Dirac cone. According to the ARPES data under various photon energies, we map out the $\mathrm{E}-\mathrm{k}_{\mathrm{z}}$ dispersions as seen in Fig. 5(b)-(d). Comparing with the calculation, ARPES dispersions are consistent with the theoretical data and the tilted Dirac cone can be recognized when $k_{x}=k_{y}=0$ (Fig. 5(b)). 
When departing from $\Gamma$-A line, as seen from Fig. 5(b) to (d), the two bands forming Dirac cone separate obviously in calculation as well as in our experimental data. One may notice that the intensity near the Dirac point is suppressed. This may explained by slight deviation from $k_{x}=k_{y}=0$. We also map out the $k_{x}-k_{z}$ constant energy contours when $\mathrm{k}_{\mathrm{y}}=0$ (Supplemental Material, Figure S2 [45]). A hole pocket and an electron pocket can be seen and tend to touch each other at Dirac point when energy goes down, which is a character of type-II Dirac dispersion. The type-II property of the Dirac cone in $\mathrm{PdTe}_{2}$ is thus confirmed.

\section{E. Discussions}

We suggest the discovered type-II Dirac semimetal be an improved platform of TSC, on which a lot of efforts are made [27, 46-50]. Recently, with the discovery of three-dimensional topological semimetal, the attempts are made on inducing the SWS in the newly discovered semimetals $[51,52]$. We here compare the TSC transport based on three kinds of mother materials, Weyl semimetal, Dirac semimetal and type-II Dirac semimetal. For the TSC arisen from the Weyl semimetals, it is known from the theoretical work $[51,52]$ that the chiral anomaly survived and invokes a Fermi arc surface state, in which the electrons remain unpaired and no gap opens. Meanwhile, the effective $\mathrm{p}_{\mathrm{x}}+\mathrm{ip} \mathrm{y}$ pairing state in the bulk leads to chiral Majorana surface mode. The coexistence of Fermi arc and the Majorana mode may hamper its application in quantum computation. For the TS arisen from Dirac semimetals, the gapless node is Dirac point which is fourfold degenerated and additional space group symmetries are required for the stability of the Dirac nodes. The Fermi arc state of the 
Dirac semimetal is not topologically stable [53]. Hence, after the superconductivity sets in, in general, the surface electrons would form Cooper pairs and gap out the surface state. The situation is improved for the case of type-II Dirac cone where the dispersion is tilted as shown in Fig. 6. The significant superiority can be seen is that the electron and hole pockets near the Dirac points provide the plenty density of states which is favorable for both superconducting and TSC carrier ratio. This also means larger carrier density in the TS arisen from type-II Dirac semimetals.

\section{CONCLUSION}

In summary, combining $\mathrm{dHvA}$ oscillations and the first-principles calculations, we verify the topological nontrivial Berry phase from the hole pocket of a tilted Dirac cone in type-II Dirac semimetal $\mathrm{PdTe}_{2}$. Angle Resolved Photoemission Spectroscopy also demonstrates the type-II Dirac dispersion. Anisotropic superconductivity below $1.9 \mathrm{~K}$ in $\mathrm{PdTe}_{2}$ is confirmed by low-temperature transport measurement. It is therefore a possible improved platform to search for mysterious Majorana Fermions and applies to the next-generation spintronics devices.

\section{ACKNOWLEDGEMENTS}

We gratefully acknowledge the financial support of the National Key Projects for Basic Research of China (Grant Nos: 2013CB922103), the National Natural Science Foundation of China (Grant Nos: 91622115, 91421109, 11574133 and 11274003), the

PAPD project, the Natural Science Foundation of Jiangsu Province (Grant 
BK20130054), and the Fundamental Research Funds for the Central Universities. Use of the Stanford Synchrotron Radiation Lightsource, SLAC National Accelerator Laboratory, is supported by the U.S. Department of Energy, Office of Science, Office of Basic Energy Sciences under Contract No. DE-AC02-76SF00515. The technical support from the Hefei National Synchrotron Radiation Laboratory and High Magnetic Field Laboratory, Chinese Academy of Sciences are acknowledged. We also thank Prof. Yongchun Tao in Nanjing Normal University, China for stimulating discussions.

\section{FOOTNOTES AND REFERENCE CITATION}

The first three authors (F. F, X. B and R. W) contributed equally to this work.

${ }^{*}$ Corresponding authors.

songfengqi@nju.edu.cn

${ }^{\dagger}$ Corresponding authors.

xgwan@nju.edu.cn

$\$$ Corresponding authors.

bgwang@nju.edu.cn

[1] Z. K. Liu, J. Jiang, B. Zhou, Z. J. Wang, Y. Zhang, H. M. Weng, D. Prabhakaran, S. K. Mo, H. Peng, P. Dudin, T. Kim, M. Hoesch, Z. Fang, X. Dai, Z. X. Shen, D. L. 
Feng, Z. Hussain, and Y. L. Chen, Nat. Mater. 13, 677 (2014).

[2] L. P. He, X. C. Hong, J. K. Dong, J. Pan, Z. Zhang, J. Zhang, and S. Y. Li, Phys. Rev. Lett. 113, 246402 (2014).

[3] H. Wang, H. Wang, H. Liu, H. Lu, W. Yang, S. Jia, X. J. Liu, X. C. Xie, J. Wei, and J. Wang, Nat. Mater. 15, 38 (2016).

[4] M. Neupane, S.-Y. Xu, R. Sankar, N. Alidoust, G. Bian, C. Liu, I. Belopolski, T.-R. Chang, H.-T. Jeng, H. Lin, A. Bansil, F. Chou, and M. Z. Hasan, Nat. Commun. 5, 3786 (2014).

[5] S. Jeon, B. B. Zhou, A. Gyenis, B. E. Feldman, I. Kimchi, A. C. Potter, Q. D. Gibson, R. J. Cava, A. Vishwanath, and A. Yazdani, Nat. Mater. 13, 851 (2014).

[6] L. He, Y. Jia, S. Zhang, X. Hong, C. Jin, and S. Li, npj Quantum Mater. 1, 16014 (2016).

[7] S. Borisenko, Q. Gibson, D. Evtushinsky, V. Zabolotnyy, B. Buchner, and R. J. Cava, Phys. Rev. Lett. 113, 027603 (2014).

[8] S.-Y. Xu, C. Liu, S. K. Kushwaha, R. Sankar, J. W. Krizan, I. Belopolski, M. Neupane, G. Bian, N. Alidoust, T.-R. Chang, H.-T. Jeng, C.-Y. Huang, W.-F. Tsai, H. Lin, P. P. Shibayev, F.-C. Chou, R. J. Cava, and M. Z. Hasan, Science 347, 294 (2015).

[9] J. Xiong, S. K. Kushwaha, T. Liang, J. W. Krizan, M. Hirschberger, W. Wang, R. J. Cava, and N. P. Ong, Science 350, 413 (2015).

[10]Z. K. Liu, B. Zhou, Y. Zhang, Z. J. Wang, H. M. Weng, D. Prabhakaran, S.-K. Mo, Z. X. Shen, Z. Fang, X. Dai, Z. Hussain, and Y. L. Chen, Science 343, 864 (2014).

[11] S.-Y. Xu, N. Alidoust*, G. Chang, H. Lu, B. Singh, I. Belopolski, D. S. Sanchez, X. Zhang, G. Bian, H. Zheng, M.-A. Husanu, Y. Bian, S.-M. Huang, C.-H. Hsu, T.-R. Chang, H.-T. Jeng, A. Bansil, V. N. Strocov, H. Lin, S. Jia, and M. Z. Hasan, arXiv: 1603.07318.

[12]L. X. Yang, Z. K. Liu, Y. Sun, H. Peng, H. F. Yang, T. Zhang, B. Zhou, Y. Zhang, Y. F. Guo, M. Rahn, D. Prabhakaran, Z. Hussain, S. K. Mo, C. Felser, B. Yan, and Y. L. Chen, Nat. Phys. 11, 728 (2015).

[13]H. M. Weng, C. Fang, Z. Fang, B. A. Bernevig, and X. Dai, Phys. Rev. X 5, 011029 (2015).

[14]N. Xu, H. M. Weng, B. Q. Lv, C. E. Matt, J. Park, F. Bisti, V. N. Strocov, D. Gawryluk, E. Pomjakushina, K. Conder, N. C. Plumb, M. Radovic, G. Autes, O. V. Yazyev, Z. Fang, X. Dai, T. Qian, J. Mesot, H. Ding, and M. Shi, Nat. Commun. 7, 11006 (2016).

[15]B. Q. Lv, H. M. Weng, B. B. Fu, X. P. Wang, H. Miao, J. Ma, P. Richard, X. C. Huang, L. X. Zhao, G. F. Chen, Z. Fang, X. Dai, T. Qian, and H. Ding, Phys. Rev. X 5, 031013 (2015).

[16] S.-Y. Xu, I. Belopolski, N. Alidoust, M. Neupane, G. Bian, C. Zhang, R. Sankar, G. Chang, Z. Yuan, C.-C. Lee, S.-M. Huang, H. Zheng, J. Ma, D. S. Sanchez, B. Wang, A. Bansil, F. Chou, P. P. Shibayev, H. Lin, S. Jia, and M. Z. Hasan, Science 349, 613 (2015).

[17]H. Zheng, S.-Y. Xu, G. Bian, C. Guo, G. Chang, D. S. Sanchez, I. Belopolski, 
C.-C. Lee, S.-M. Huang, X. Zhang, R. Sankar, N. Alidoust, T.-R. Chang, F. Wu, T. Neupert, F. Chou, H.-T. Jeng, N. Yao, A. Bansil, S. Jia, H. Lin, and M. Z. Hasan, ACS Nano 10, 1378 (2016).

[18]Y. Qi, P. G. Naumov, M. N. Ali, C. R. Rajamathi, W. Schnelle, O. Barkalov, M. Hanfland, S. C. Wu, C. Shekhar, Y. Sun, V. Suss, M. Schmidt, U. Schwarz, E. Pippel, P. Werner, R. Hillebrand, T. Forster, E. Kampert, S. Parkin, R. J. Cava, C. Felser, B. Yan, and S. A. Medvedev, Nat. Commun. 7, 11038 (2016).

[19]L. Huang, T. M. McCormick, M. Ochi, Z. Y. Zhao, M. T. Suzuki, R. Arita, Y. Wu, D. X. Mou, H. B. Cao, J. Q. Yan, N. Trivedi, and A. Kaminski, Nat. Mater. 15, 1155 (2016).

[20]X. C. Pan, X. Chen, H. Liu, Y. Feng, Z. Wei, Y. Zhou, Z. Chi, L. Pi, F. Yen, F. Song, X. Wan, Z. Yang, B. Wang, G. Wang, and Y. Zhang, Nat. Commun. 6, 7805 (2015).

[21]Z. J. Wang, D. Gresch, A. A. Soluyanov, W. W. Xie, S. Kushwaha, X. Dai, M. Troyer, R. J. Cava, and B. A. Bernevig, Phys. Rev. Lett. 117, 056805 (2016).

[22]I. Belopolski, S. Y. Xu, Y. Ishida, X. C. Pan, P. Yu, D. S. Sanchez, H. Zheng, M. Neupane, N. Alidoust, G. Q. Chang, T. R. Chang, Y. Wu, G. Bian, S. M. Huang, C. C. Lee, D. X. Mou, L. N. Huang, Y. Song, B. G. Wang, G. H. Wang, Y. W. Yeh, N. Yao, J. E. Rault, P. Le Fevre, F. Bertran, H. T. Jeng, T. Kondo, A. Kaminski, H. Lin, Z. Liu, F. Q. Song, S. Shin, and M. Z. Hasan, Phys. Rev. B 94, 085127 (2016).

[23]I. Belopolski, D. S. Sanchez, Y. Ishida, X. Pan, P. Yu, S. Y. Xu, G. Chang, T. R. Chang, H. Zheng, N. Alidoust, G. Bian, M. Neupane, S. M. Huang, C. C. Lee, Y. Song, H. Bu, G. Wang, S. Li, G. Eda, H. T. Jeng, T. Kondo, H. Lin, Z. Liu, F. Song, S. Shin, and M. Z. Hasan, Nat. Commun. 7, 13643 (2016).

[24]Y. Xu, F. Zhang, and C. Zhang, Phys. Rev. Lett. 115, 265304 (2015).

[25]L. Fu and C. L. Kane, Phys. Rev. Lett. 100, 096407 (2008).

[26]J. J. He, T. K. Ng, P. A. Lee, and K. T. Law, Phys. Rev. Lett. 112, 037001 (2014).

[27]H.-H. Sun, K.-W. Zhang, L.-H. Hu, C. Li, G.-Y. Wang, H.-Y. Ma, Z.-A. Xu, C.-L. Gao, D.-D. Guan, Y.-Y. Li, C. Liu, D. Qian, Y. Zhou, L. Fu, S.-C. Li, F.-C. Zhang, and J.-F. Jia, Phys. Rev. Lett. 116, 257003 (2016).

[28]J. P. Xu, M. X. Wang, Z. L. Liu, J. F. Ge, X. Yang, C. Liu, Z. A. Xu, D. Guan, C. L. Gao, D. Qian, Y. Liu, Q. H. Wang, F. C. Zhang, Q. K. Xue, and J. F. Jia, Phys. Rev. Lett. 114, 017001 (2015).

[29]B. Bradlyn, J. Cano, Z. Wang, M. G. Vergniory, C. Felser, R. J. Cava, and B. A. Bernevig, Science 353, 6299 (2016).

[30]S. Zhang, L. Pi, R. Wang, G. Yu, X.-C. Pan, Z. Wei, J. Zhang, C. Xi, Z. Bai, F. Fei, M. Wang, J. Liao, Y. Li, X. Wang, F. Song, Y. Zhang, B. Wang, D. Xing, and G. Wang, arXiv: 1702.03344.

[31] T. Chen, Q. Chen, K. Schouteden, W. Huang, X. Wang, Z. Li, F. Miao, X. Wang, Z. Li, B. Zhao, S. Li, F. Song, J. Wang, B. Wang, C. Van Haesendonck, and G. Wang, Nat. Commun. 5, 5022 (2014).

[32] T. Chen, W. Liu, F. Zheng, M. Gao, X. Pan, G. van der Laan, X. Wang, Q. Zhang, F. Song, B. Wang, B. Wang, Y. Xu, G. Wang, and R. Zhang, Adv. Mater. 27, 4823 (2015). 
[33]F. Fei, Z. Wei, Q. Wang, P. Lu, S. Wang, Y. Qin, D. Pan, B. Zhao, X. Wang, J. Sun, X. Wang, P. Wang, J. Wan, J. Zhou, M. Han, F. Song, B. Wang, and G. Wang, Nano Lett. 15, 5905 (2015).

[34]M. Udagawa and E. J. Bergholtz, Phys. Rev. Lett. 117, 086401 (2016).

[35]T. E. O'Brien, M. Diez, and C. W. Beenakker, Phys. Rev. Lett. 116, 236401 (2016).

[36]H. Huang, S. Zhou, and W. Duan, Phys. Rev. B 94, 121117(R) (2016).

[37] M. Yan, H. Huang, K. Zhang, E. Wang, W. Yao, K. Deng, G. Wan, H. Zhang, M. Arita, H. Yang, Z. Sun, H. Yao, Y. Wu, S. Fan, W. Duan, and S. Zhou, arXiv: 1607.03643 .

[38]H.-J. Noh, J. Jeong, and E.-J. Cho, arXiv: 1612.06946.

[39]A. E. Dunsworth, J. Low Temp. Phys. 19, 51 (1975).

[40]v. J. Guggenheim and F. H. u. J. Müller, H. P. A. 34, 408 (1961).

[41]I. M. Lifshitz and A. M. Kosevich, Sov. Phys. JETP 2, 636 (1956).

[42] J. Hu, Z. Tang, J. Liu, X. Liu, Y. Zhu, D. Graf, K. Myhro, S. Tran, C. N. Lau, J. Wei, and Z. Mao, Phys. Rev. Lett. 117, 016602 (2016).

[43]Y. Liu, J.-Z. Zhao, L. Yu, C.-T. Lin, A.-J. Liang, C. Hu, Y. Ding, Y. Xu, S.-L. He, L. Zhao, G.-D. Liu, X.-L. Dong, J. Zhang, C.-T. Chen, Z.-Y. Xu, H.-M. Weng, X. Dai, Z. Fang, and X.-J. Zhou, Chinese Phys. Lett. 32, 067303 (2015).

[44]A. A. Soluyanov, D. Gresch, Z. Wang, Q. Wu, M. Troyer, X. Dai, and B. A. Bernevig, Nature 527, 495 (2015).

[45] See Supplemental Material at [] for detailed information of crystal growth and characterization, method of electronic structure calculations, ARPES measurement at various photon energies.

[46] S.-Y. Xu, N. Alidoust, I. Belopolski, A. Richardella, C. Liu, M. Neupane, G. Bian, S.-H. Huang, R. Sankar, C. Fang, B. Dellabetta, W. Dai, Q. Li, M. J. Gilbert, F. Chou, N. Samarth, and M. Z. Hasan, Nat. Phys. 10, 943 (2014).

[47]M.-X. Wang, C. Liu, J.-P. Xu, F. Yang, L. Miao, M.-Y. Yao, C. L. Gao, C. Shen, X. Ma, X. Chen, Zhu-An, X. Liu, S.-C. Zhang, D. Qian, J.-F. Jia, and Q.-K. Xue, Science 336, 52 (2012).

[48] S. Yonezawa, K. Tajiri, S. Nakata, Y. Nagai, Z. Wang, K. Segawa, Y. Ando, and Y. Maeno, Nat. Phys., doi:10.1038/nphys3907.

[49] Y. S. Hor, A. J. Williams, J. G. Checkelsky, P. Roushan, J. Seo, Q. Xu, H. W. Zandbergen, A. Yazdani, N. P. Ong, and R. J. Cava, Phys. Rev. Lett. 104, 057001 (2010).

[50]Z. Liu, X. Yao, J. Shao, M. Zuo, L. Pi, S. Tan, C. Zhang, and Y. Zhang, Journal of the American Chemical Society 137, 10512 (2015).

[51]G. Bednik, A. A. Zyuzin, and A. A. Burkov, Phys. Rev. B 92, 035153 (2015).

[52]R. Wang, L. Hao, B. Wang, and C. S. Ting, Phys. Rev. B 93, 184511 (2016).

[53] M. Kargarian, M. Randeria, and Y. M. Lu, arXiv: 1509.02180. 


\section{FIGURE CAPTIONS}

FIG. $1 \mathrm{PdTe}_{2}$ Crystal growth and characterization. (a) The $\mathrm{CdI}_{2}$-type crystal structure of $\mathrm{PdTe}_{2}$ and the corresponding Brillouin zone (lower right panel) (b) The X-ray diffraction data of $\mathrm{PdTe}_{2}$ sample. Strong (00n) peaks can be seen. The inset is optical

micrograph of several flakes. (c) The EDS spectrum indicates stoichiometric ratio of the sample. The insets show the EDS mapping of Pd and Te, respectively, of a typical flake. (d) Raman spectrum of $\mathrm{PdTe}_{2}$ crystal.

FIG. 2 Electrical transport under magnetic fields and low temperature. (a) The magnetoresistance of $\mathrm{PdTe}_{2}$ under $1.7 \mathrm{~K}$. The inset shows $\mathrm{SdH}$ oscillation after background subtraction. (b) Hall resistance versus magnetic field under various temperatures. (c) and (d) are respectively temperature dependence of resistance under different fields of configuration $1(\mathrm{I} \perp \mathrm{c}, \mathrm{B} / / \mathrm{c})$ and $2(\mathrm{I} / / \mathrm{c}, \mathrm{B} / / \mathrm{c})$, indicating anisotropic superconductivity in $\mathrm{PdTe}_{2}$.

FIG. 3. The dHvA oscillations and nontrivial Berry phase. (a) The magnetization curve $(\mathrm{B} / / \mathrm{c})$ for $\mathrm{PdTe}_{2}$ at $1.8 \mathrm{~K}$. Beautiful dHvA oscillations can be seen. (b) The magnetization strength versus 1/B under various temperatures after background subtraction. (c) The fits of effective mass for all six oscillation modes ( $\alpha$ to $\zeta)$. The inset is the FFT amplitudes versus temperature. Peak corresponding to each oscillation mode was marked by Greek letter. (d) The Landau fan diagram for the 
dHvA oscillation of low frequency mode $\alpha$. The inset is the fit of Dingle temperature $\left(T_{D}\right)$. (e) Oscillatory component of other frequencies $(\beta$ to $\zeta)$ and the multiband LK fit of it. Inset shows a part of the curve, indicating the perfect fit of the experimental data.

FIG. 4. Matching the dHvA components in the calculations of type-II Dirac cone. (a) The calculated electronic structures plotted along the directions shown in Brillouin zone (Fig. 1(a)). The Dirac point and the small hole pocket with $\alpha$ mode is marked in the panel. (b) and (c) show the contour of Fermi surface along $\mathrm{k}_{\mathrm{x}}-\mathrm{k}_{\mathrm{y}}$ and $\mathrm{k}_{\mathrm{x}}-\mathrm{k}_{\mathrm{z}}$ plane respectively. Pockets irrelevant to the hole and electron pockets form by the tilted Dirac cone in (c) are concealed for clearness. (d) and (e) show the projection of the Dirac cone along $\mathrm{k}_{\mathrm{x}}-\mathrm{k}_{\mathrm{y}}$ and $\mathrm{k}_{\mathrm{x}}-\mathrm{k}_{\mathrm{z}}$ respectively. Dirac cone with tilted properties along $\mathrm{k}_{\mathrm{z}}$ direction can be clearly seen.

FIG. 5. ARPES measurement of $\mathrm{PdTe}_{2}$. (a) The dispersion along T-D direction measured by ARPES. The linear dispersion of Dirac cone is labeled by the red dash lines. The Dirac point is also marked by the red arrow. (b)-(d) are dispersions along $\mathrm{k}_{\mathrm{z}}$ directions. Black dash lines are band calculations for comparison.

FIG. 6. Optimizing the TSC by type-II Dirac semimetals. This figure displays the band structure diagrams of the possible topological superconductors based on the mother materials of type-II Dirac semimetal when $\mathrm{T}<\mathrm{T}_{\mathrm{c}}$. The red solid cross curves in 
superconducting (SC) gap (grey zone) represent the Majorana chiral mode. The Fermi arc states are killed in Dirac semimetals when the superconductivity occurs. 


\section{FIGURES}

(a)

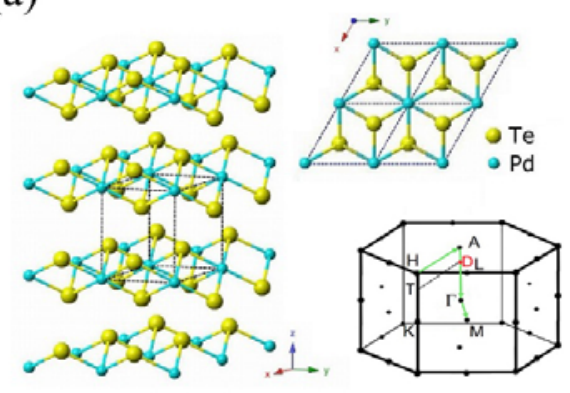

(c)

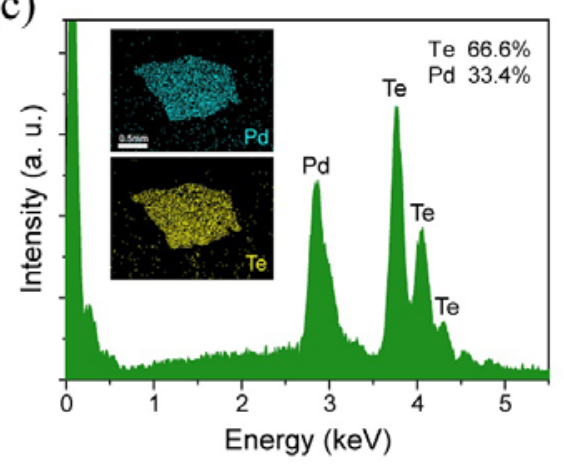

(b)

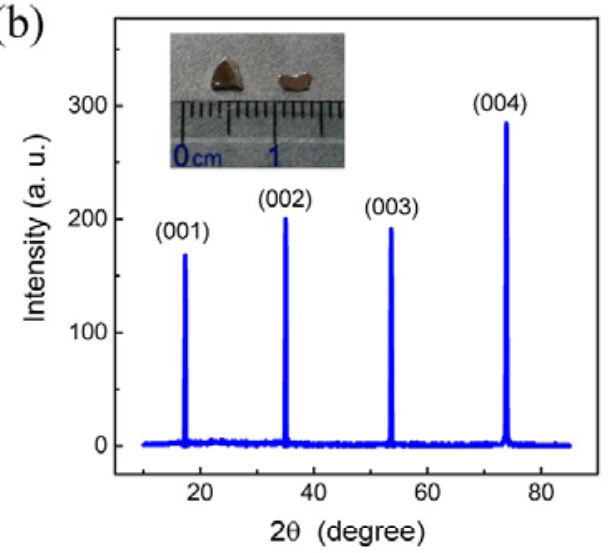

(d)

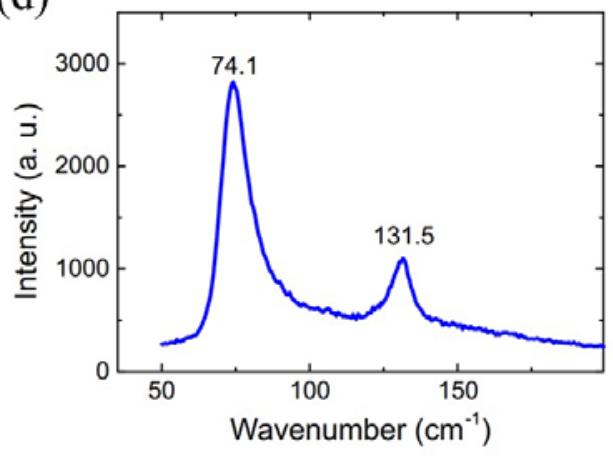

FIG. 1. $\mathrm{PdTe}_{2}$ Crystal growth and characterization. 
(a)

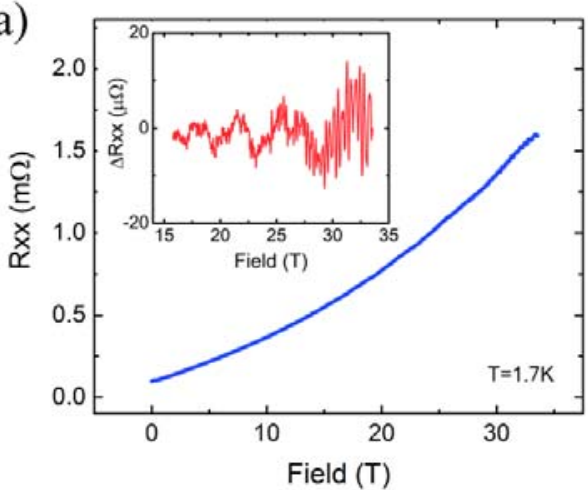

(c)

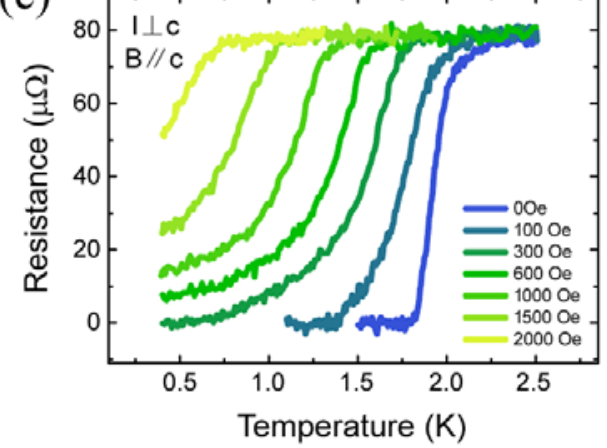

(b)

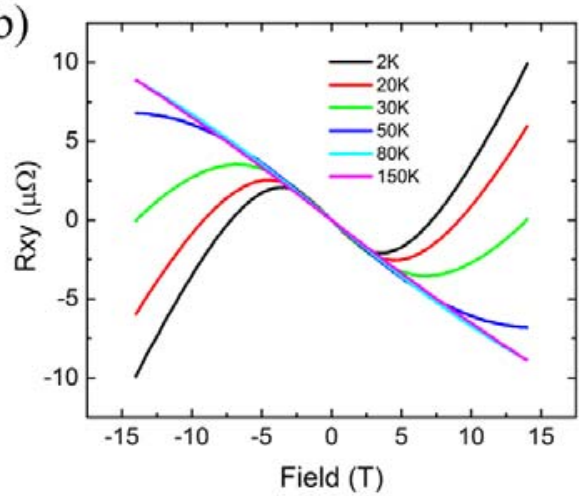

(d)

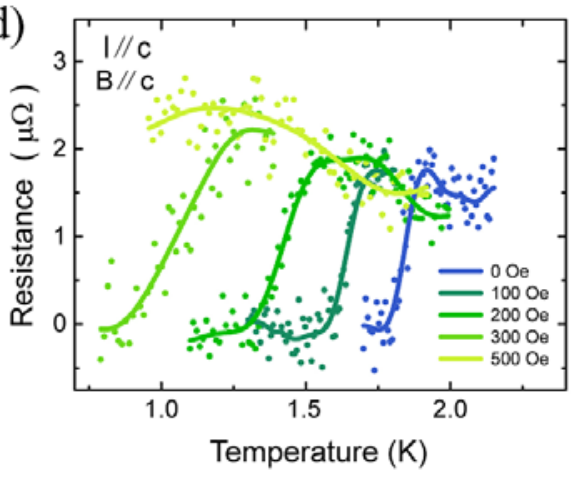

FIG. 2. Electrical transport under magnetic fields and low temperature. 

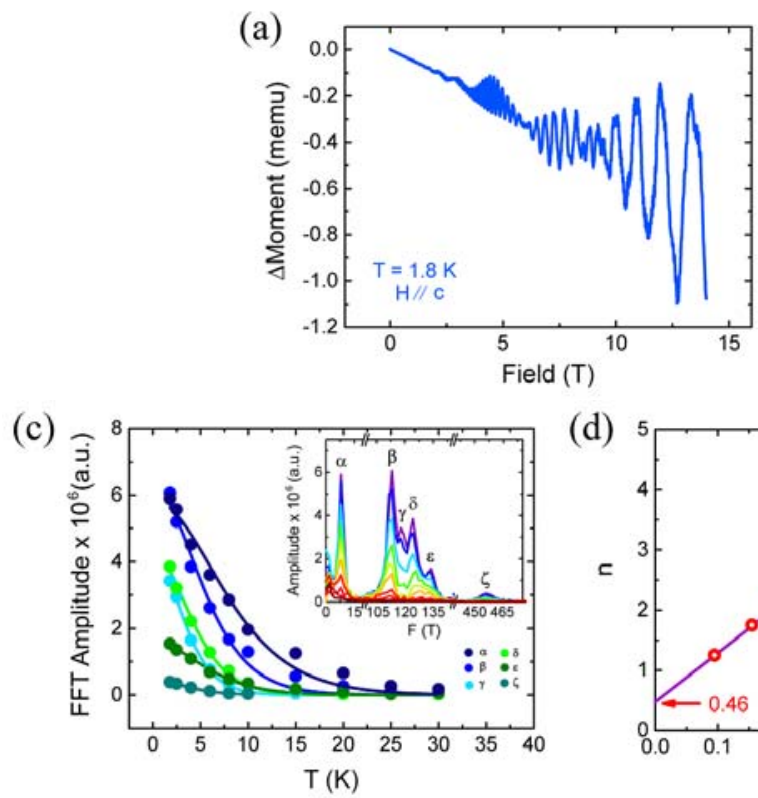

(d)
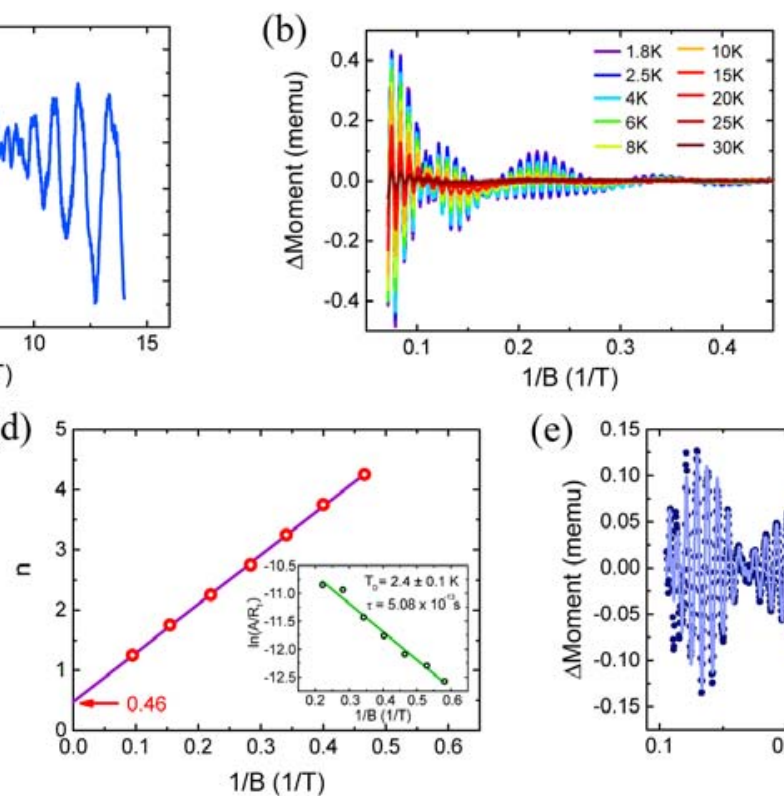

(e)

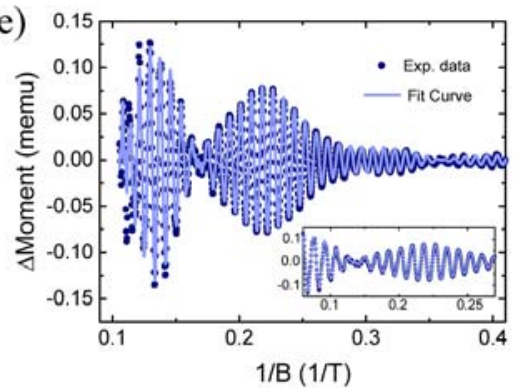

FIG. 3. The dHvA oscillations and nontrivial Berry phase. 


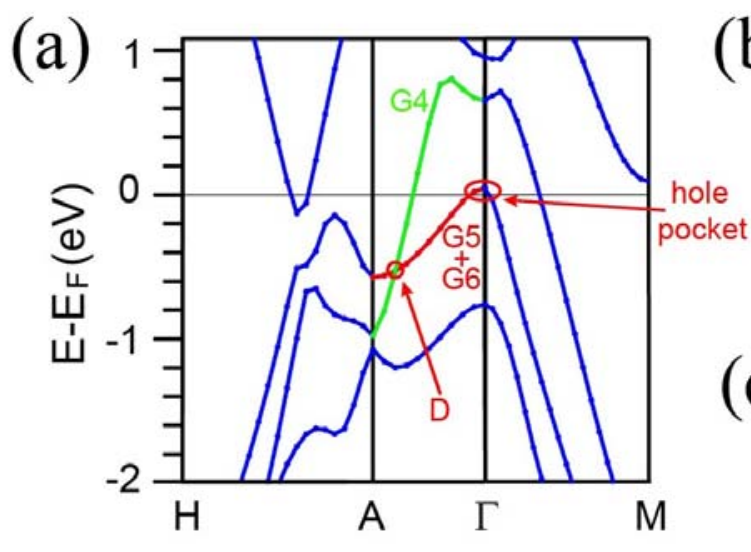

(b)

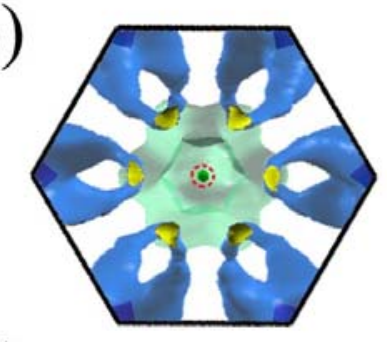

(c)
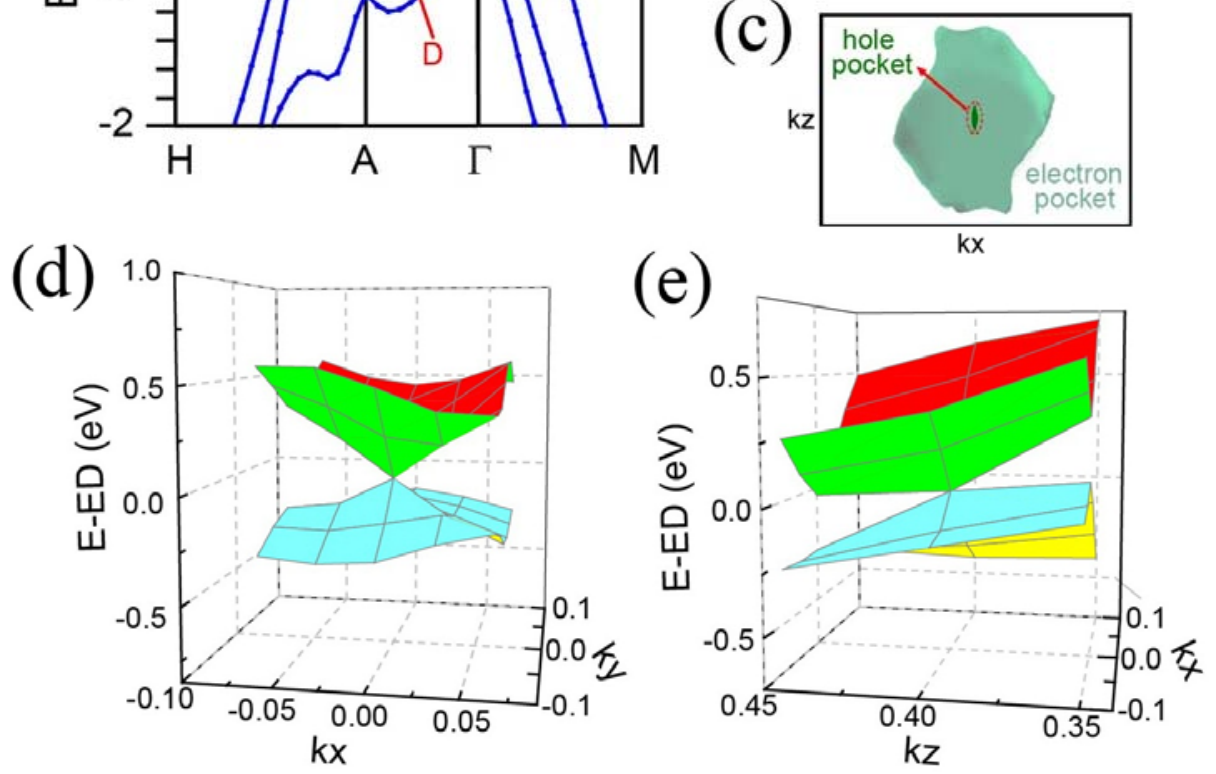

FIG. 4. Matching the dHvA components in the calculations of type-II Dirac cone. 

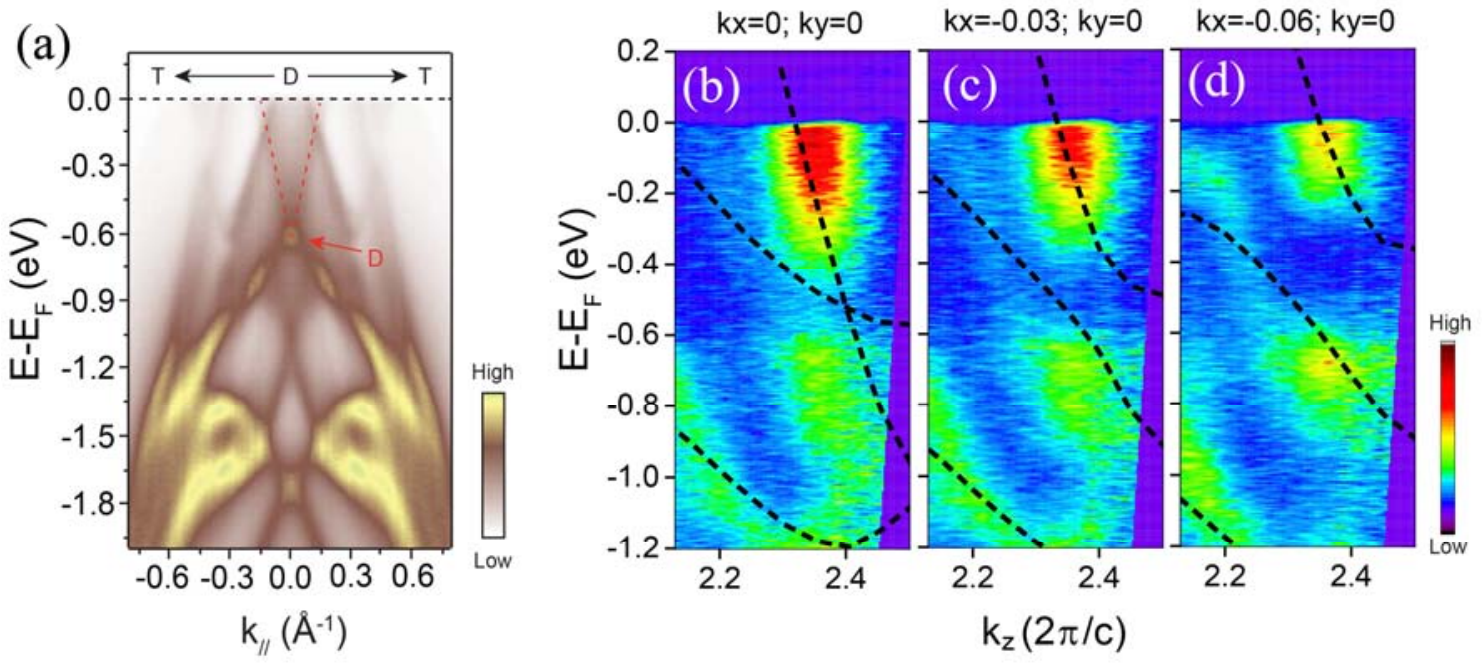

FIG. 5. ARPES measurement of $\mathrm{PdTe}_{2}$. 


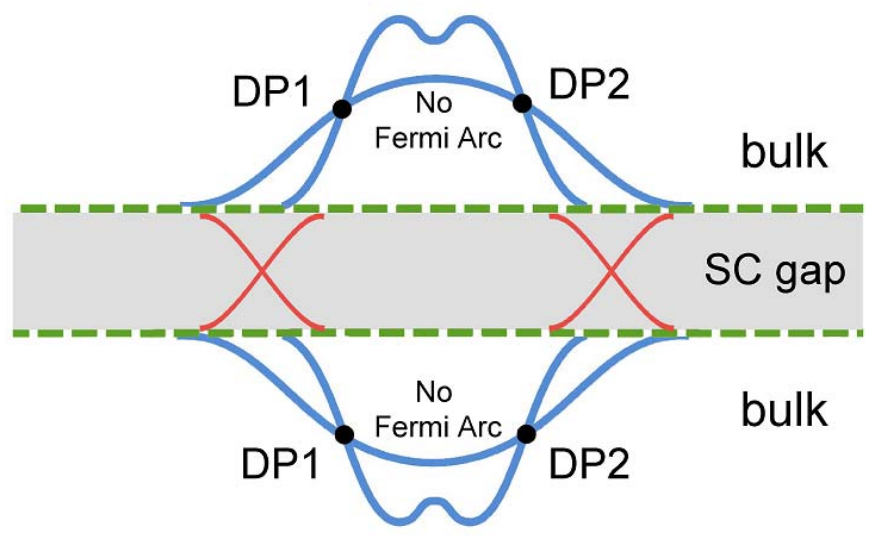

FIG. 6. Optimizing the TSC by type-II Dirac semimetals. 


\section{TABLES}

TABLE I. Parameters extracted from the dHvA oscillations $(\mathrm{H} / / \mathrm{c})$ of $\mathrm{PdTe}_{2} . F$, oscillation frequency; $S_{f}$, crosssection of Fermi surface; $k_{f}$, Fermi wave vector; $T_{D}$, Dingle temperature; $m * / m_{0}$, relative effective mass; $\tau$, quantum relaxation time; $\mu_{q}$, quantum mobility.

\begin{tabular}{ccccccc}
\hline \hline$F(T)$ & $S_{f}\left(\AA^{-2}\right)$ & $k_{f}\left(\AA^{-1}\right)$ & $T_{D}(K)$ & $m^{*} / m_{0}$ & $\tau(p s)$ & $\mu_{q}\left(\mathrm{~cm}^{2} / V s\right)$ \\
\hline 8.0 & $7.67 \times 10^{-4}$ & $1.56 \times 10^{-2}$ & 2.4 & 0.14 & 0.51 & 6209 \\
113.2 & $1.09 \times 10^{-2}$ & $5.88 \times 10^{-2}$ & 3.4 & 0.21 & 0.36 & 3030 \\
117.9 & $1.13 \times 10^{-2}$ & $6.00 \times 10^{-2}$ & 1.9 & 0.33 & 0.65 & 3464 \\
124.3 & $1.19 \times 10^{-2}$ & $6.16 \times 10^{-2}$ & 3.0 & 0.26 & 0.41 & 2722 \\
133.9 & $1.28 \times 10^{-2}$ & $6.39 \times 10^{-2}$ & 6.8 & 0.20 & 0.18 & 1593 \\
455.8 & $4.34 \times 10^{-2}$ & 0.118 & 5.7 & 0.29 & 0.21 & 1293 \\
\hline \hline
\end{tabular}

